\title{
Clinical Governance: Learning and changing practice
}

\author{
Sue Ingram, RGN, Clinical Nurse Specialist; Sarah Randall, MD, MFFP, FRCOG, Consultant Reproductive Health, Ella Gordon \\ Unit, St Mary's Hospital, Portsmouth, UK.
}

Correspondence: Dr Sarah Randall, Ella Gordon Unit, St Mary’s Hospital, Portsmouth, Hampshire, PO3 6AD, UK

(Accepted 25 $5^{\text {th }}$ June 2001)

The Journal of Family Planning and Reproductive Health Care 2001: 27(4): 228-230

\begin{abstract}
Context. This paper describes a very simple, nonthreatening method for improving communication and sharing learning points.

Objective. To test whether sharing anonymised reporting of problems and helpful hints is acceptable and useful to staff. Design. A pink slip (pinkie) was designed and made available in every clinic venue. All staff were asked to write about any episodes where practice was less than optimum or to share good clinical experiences. The forms could be completed anonymously if preferred. A senior nurse collated the reports monthly and fed them back to all staff as a report. In May 2000 all staff were surveyed for their opinion of the scheme.
\end{abstract}

Result. Over 22 months, 139 'pinkies' were returned. Fiftysix of the 100 'less than optimum' events were classified as 'system failures'. The response to the staff survey was very positive, with $62 \%$ of staff replying. Fifty-four of the 55 staff found the scheme helpful. A third of those who responded had contributed and all but two individuals felt able to contribute if the situation arose again.

Conclusion. This simple system of self completed pink slips has allowed examples of less than optimum practice and helpful suggestions to be shared across a large service that has a predominantly part time work force providing services from over 15 venues. The system is seen as nonthreatening and was acceptable to over $95 \%$ of the staff who responded to the survey. Fifty percent of doctors and nurses had made a submission. Changes in practice have resulted since its introduction.

\section{Key message points}

- A simple system whereby we can all learn from our, and others', problems and helpful suggestions.

- Anonymised reporting is acceptable to all grades of staff.

- This mechanism was seen as a non-threatening and innovative way of learning.

\section{Introduction}

The Ella Gordon Unit is the main centre of a large community-based contraception and sexual health service in the Portsmouth and South East Hampshire Health Authority. The area covers a population of 500000 . In 1999 the service, which runs in excess of 70 clinics per week, received nearly 60000 visits and is one of the largest in the country. As well as providing a comprehensive contraceptive service from 10 sites and five venues for the under $25 \mathrm{~s}$, it also runs the district termination service and provides specialist advice in a variety of areas including the menopause.
Our main problem is one of communication and sharing good practice. Many hospital specialties have mainly full time staff working from a single base. This makes it easier to arrange staff meetings. In community-based family planning (FP) clinics staff work mainly part-time, and many have other jobs and service provision is from multiple venues. Communication has therefore to be by post or email. We have a robust system of sending out guidelines etc, but staff may not always read everything that arrives. We therefore wanted to look at different ways of alerting staff to new situations.

In early 1999, the obstetric and gynaecology department in Portsmouth introduced a system of logging 'risk' events which included, for example when patients had to go back to theatre within 24 hours of the original operation or when a post partum haemorrhage occurred. These forms were reviewed monthly and action taken as required.

We thought that such a scheme could be used to good effect in our department as a part of clinical governance. ${ }^{1,2}$ It was felt that using this system of sharing potential problems and good ideas was a way of encouraging all staff to question their own practice and keep themselves updated. It started in June 1999.

\section{Method}

All staff were informed about the scheme. We made it very clear that serious adverse events and formal complaints should be dealt with as before according to the Trust protocol. We also continued to undertake formal audit on such topics as re-admission following termination of pregnancy (TOP) and vasectomy failure.

What we asked them to write about were episodes where practice was less than optimum: problems with day to day management of results or notes where it was felt improvement could take place and to share good clinical experiences that might be of use to other clinicians.

The system works using pink slips. These are available in all clinic areas including reception. Any staff member can access a form very easily and can complete it anonymously if they wish. We ask for details of the event and the outcome. Patient details are not included to maintain their confidentiality. Ideally we like to know which staff were involved (although this is not mandatory), when the event occurred, and the clinic venue. The forms are sent in confidence to the senior nurse. She reviews them monthly and transcribes them (so that handwriting cannot be recognised) onto a 'risk assessment' sheet, anonymising them if needs be. The events are written up each month and circulated to all staff.

In May 2000 we wrote to all regular staff to gauge their opinion of the scheme. 


\section{Results}

Over the past 22 months, 139 'pinkies' have been returned. The forms are classified according to the type of health professional (if known) i.e. medical, nursing, clerical, counsellor, project worker and also as to whether it was a 'less than optimum' event or a 'helpful suggestion' or 'risk averted'. The results are shown in Table 1. The table shows that there were 139 responses, with 100 being classified as 'less than optimum' events. Fifty-six of the 100 'less than optimum events' could also have been classified as 'system failures', with staff not being aware of procedures. Examples are listed in Table 2.

Table 1 Classification of event type by health care professional

\begin{tabular}{lcc}
\hline Staff group & $\begin{array}{c}\text { Less than } \\
\text { optimum event }\end{array}$ & $\begin{array}{c}\text { Helpful suggestion / } \\
\text { Risk averted }\end{array}$ \\
\hline Medical & 51 & 13 \\
Nursing & 36 & 21 \\
Clerical & 13 & 5 \\
Other / not stated & 0 & 0 \\
Total & 100 & 39 \\
\hline
\end{tabular}

The results of the survey to assess staff response to the 'pinkies' are shown in Table 3.

\section{Discussion}

Communication between staff in a service that has mainly part timers who work from many different venues is not easy, as it is virtually impossible to get them all together in one place at one time. Alternative methods of sharing information have to be used. The Trust and service monthly 'information exchanges' are used, and regular mailings sent out about changes to protocols, new systems etc. But we all suffer from information overload. We wanted a simple system that would attract the staff's attention and that they would enjoy reading.

The simple system of 'pinkies' seems to be working. Fifty percent of doctors and nurses have submitted a contribution.

We have now extended their use in two other areas. Firstly, to collect data on intra-uterine devices (IUDs). Any IUD event is written up and sent to one of the authors. This may include a failed fitting and reasons for removal.

The second use has resulted because we had a problem with men attending for a vasectomy under local anaesthesia having to be turned away because they actually needed a general anaesthetic. We circulated all GPs and FP doctors and nurses who undertake vasectomy counselling and advised that if they examined the patient and were unsure as to whether they were fit for local anaesthesia that they should refer them for a pre-operative assessment. Since we have done this the number of 'refused' operations has decreased. The 'pink' slips allow us to monitor these and any further problems.

\section{Conclusion}

This is a simple system that has been shown to be acceptable, non-threatening and effective in communicating 'near misses' and 'successes' to a large work force who work mainly part time in many different venues. It could easily be adapted to fit other services' needs.

Table 2 Examples of 'pinkies' including those that have resulted in a change of policy

\begin{tabular}{lll}
\hline Staff Member & Problem & Reason \\
Medical & Oral EC not given at first visit. & $\begin{array}{l}\text { Patient decided to have IUD fitted } \\
\text { the next day but then at the second } \\
\end{array}$ \\
& & visit changed her mind. Oral EC \\
& was then given.
\end{tabular}

Medical Scan report stating ? ectopic pregnancy not actioned immediately it arrived.

Chlamydia swab is positive an (system failure) patient did not return to clinic.

Nursing Patient seen by domiciliary nurse 4 weeks late for Noristerat injection. (12 weekly) but attended clinic

Clerical Test result not available when patient attended for follow up.

Clerical

Clerk did not use mute button

(system failure) on telephone.

\section{Staff Member Helpful suggestion}

Medical 'pinkie' stating that a patient on injectable had lost a lot of weight and had checked her urine \& found sugar.
Doctor on holiday and result left in her pigeon hole. Nurse checked and found result.

Notes state 'no correspondence' and no other contact details obtained. imperative if a test is undertaken. with problems and was changed to Noristerat (8 weekly). Staff did not inform domiciliary nurse of change of injection.

\section{Action}

Procedure changed to ensure all patients get EC at first visit even if they decide to have an IUD fitted later.

Two actions:

a] All staff have been asked to nominate a 'buddy' when they go on holiday to deal with urgent post.

b] Senior nurse reviews all results every lunchtime and actions as necessary.

All staff reminded that a contact number / address is

All clinic staff reminded to keep domiciliary nurses informed of any of their patients who attend clinic especially if management changes have occurred.

Result had not been received from the lab and clerical staff had not noticed it was missing and had not chased it up. It was a positive Chlamydia result.

Client could overhear conversations about other patients. 
